\title{
On the traces of Hecke operators for a normalizer of $\boldsymbol{\Gamma}_{0}(\boldsymbol{N})$
}

\author{
By
}

\author{
Masatoshi YAMAUCHI
}

(Received, August 30, 1972)

For a positive integer $N$, let $\Gamma_{0}(N)$ be the congruence subgroup of level $N$, i.e. $\Gamma_{0}(N)=\left\{\left(\begin{array}{ll}a & c \\ b & d\end{array}\right) \in \mathrm{SL}_{2}(\mathbf{Z}) \mid c \equiv 0 \bmod N\right\}$ and $S_{k}(N)$ denote the space of cusp forms $f$ of weight $k$ for $\Gamma_{0}(N)$. Let $q$ be a prime divisor of $N$ and $q^{v} \| N$. Let $W_{q^{\nu}}$ denote an element of the order $R=$ $\left(\begin{array}{ll}\mathbf{Z} & \mathbf{Z} \\ N \mathbf{Z} & \mathbf{Z}\end{array}\right)$ of the form $W_{q^{\nu}}=\left(\begin{array}{ll}q^{v} x & y \\ N z & q^{v} w\end{array}\right)$ such that det $W_{q^{\nu}}=q^{v}$ with integers $x, y, z$ and $w$. In other words, putting $R_{q}=\underset{\mathbf{Z}}{\otimes} \mathbf{Z}_{q}, W_{q^{\nu}}$ denote any one of the elements of $R^{\times} \cap\left(\begin{array}{lr}0 & -1 \\ q^{v} & 0\end{array}\right) R_{q}^{\times}$, where $R^{\times}$(resp. $\left.R_{q}^{\times}\right)$is the group of all units in $R\left(\right.$ resp. $R_{q}$ ). Then $W_{q^{\nu}}$ normalizes $\Gamma_{0}(N)$ and keeps $S_{k}(N)$ invariant under the usual operation $f \mapsto f \mid W_{q^{\nu}}$ (see 1.1). Recently, $\mathrm{H}$. Hijikata [2] has given the trace formula of the Hecke operator $T_{n}$ acting on $S_{k}(N)$ for arbitrary $N$. Now the purpose of this paper is to give the trace of the operator $T_{n} \cdot W_{q^{\nu}}$ on the space $S_{k}(N)$. Then using the result of [2], we are able to give the traces of Hecke operators on the space of cusp forms for a normalizer of $\Gamma_{0}(N)$, generated by $\Gamma_{0}(N)$ and $W_{q^{\nu}}$. We mainly follow the terminology and notation by [2].

1.1. For a complex valued function $f(z)$, difine the operator

$$
f \mid \sigma=(\operatorname{det} \sigma)^{k / 2} \cdot(c z+d)^{-k} f\left(\frac{a z+b}{c z+d}\right)
$$


where $\sigma=\left(\begin{array}{ll}a & c \\ b & d\end{array}\right) \in G L_{2}(\mathbf{R})$, and $k$ is a positive integer. We denote the space of cusp forms of weight $k$ for $\Gamma_{0}(N)$ by $S_{k}(N)$, i.e. the set of all holomorphic function $f(z)$ on the complex upper half plane satisfying $f \mid \sigma=f$ for any $\sigma \in \Gamma_{0}(N)$ and vanishes at every cusps of $\Gamma_{0}(N)$. Let $n$ be a positive integer prime to $N$, then the Hecke operator $T_{n}$ is acting on $S_{k}(N)$ by

$$
f \circ T_{n}=n^{k / 2-1} \Sigma f \mid \beta_{j}
$$

where $T_{n}$ corresponds to the union of the double cosets $\Xi=\cup \Gamma \alpha_{i} \Gamma$ and we put its left cosets $\Xi=\cup \Gamma \beta_{j}$ with $\Gamma=\Gamma_{0}(N)$, or explicitly

$$
f \circ T_{n}=n^{k-1} \sum_{\substack{a d=n \\ a>0}} \sum_{b \bmod d} f\left(\frac{a z+b}{d}\right) d^{-k} .
$$

1.2. Let $N=\prod_{i=1}^{t} q_{i}^{{ }^{i}}$ where $q_{i}$ are distinct primes and let $N_{0}=\prod_{i=1}^{u} q_{i}^{\nu_{i}}$ where $0<u \leqq t$. For $q_{i} \mid N_{0}$, we define $W_{q_{i}{ }^{{ }^{i}}}=\left(\begin{array}{ll}q_{i}^{v^{i}} x & y \\ N z & q_{i}^{{ }^{i}}{ }^{i} w\end{array}\right)$ where $x, y, z$

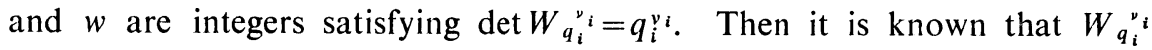
defines a C-linear automorphism of order 2: $f \mapsto f \mid W_{q_{i}{ }^{{ }}{ }^{i}}$ on $S_{k}(N)$, moreover $W_{q_{i}}^{v_{i}}$ commutes with $T_{n}$ ([1, Lemma 17]). Define

$$
W_{N_{0}}=\prod_{q \mid N_{0}} W_{q^{\nu}}
$$

1.3. Since $\Gamma W_{N_{0}} \Gamma=\Gamma W_{N_{0}}=W_{N_{0}} \Gamma$ and $T_{n}$ corresponds to the union of the double cosets $\Xi=\cup \Gamma \alpha_{i} \Gamma$, the operator $T_{n} \cdot W_{N_{0}}$ corresponds to $\Xi W_{N_{0}}=\cup \Gamma \alpha_{i} W_{N_{0}} \Gamma$. Thus the trace $\operatorname{tr} T_{n} W_{N_{0}}$ is given as follows by the formula of Hijikata ([2, Theorem $0.1,5.4]$ )

$$
\operatorname{tr} T_{n} W_{N_{0}}=-\sum_{s} a(s) \sum_{f} b(s, f) \prod_{q \mid N} c^{*}(s, f, q)+\delta(k) \cdot \sum_{\left.d\right|_{n}} d,
$$

where $\delta(k)=1$ or 0 according as $k=2$ or not. The meaning of the symbols $a(s), b(s, f)$ etc. shall be given in the following. Let $s$ run over all integers such that

$$
s^{2}-4 n N_{0}= \begin{cases}0 & \text { case }(p) \\ t^{2} & \text { case }(h)\end{cases}
$$




$$
\left.\mid \begin{array}{lll}
t^{2} m & m \equiv 1 & \bmod 4 \\
t^{2} \cdot 4 m & m \equiv 2,3 & \bmod 4
\end{array}\right\} \text { case }(e)
$$

with a positive integer $t$ and a negative square free $m$. Corresponding to the above case of $s, a(s)$ is given by

$$
a(s)=\left\{\begin{array}{l}
\frac{|x|}{4} \\
\min \{|x|,|y|\}^{k-1} \cdot\left(n N_{0}\right)^{1-k / 2} \\
\frac{1}{2}\left(x^{k-1}-y^{k-1}\right)(x-y)^{-1} \cdot\left(n N_{0}\right)^{1-k / 2}
\end{array}\right.
$$

where $x$ and $y$ are the solutions of the equation $\Phi_{s}(X)=X^{2}-s X+$ $n N_{0}=0$. For each $s$ fixed, let $f$ run over all positive divisors of $t$ in the cases $(h)$ and $(e)$, and $f=1$ in the case $(p)$. Let $K$ be the quotient ring $\mathrm{Q}[X] / \Phi_{s}(X)$ and $g$ denote the canonical image of $X$ in $K$. $K$ is a commutative Q-algebra of rank 2 , and $g$ generates an order $\mathbf{Z}+\mathbf{Z} g$ in $K$. Put $\Delta=\left(s^{2}-4 n N_{0}\right) / f^{2}$, then for each $f$, there is a uniquely determined order $\Lambda=\Lambda_{\Delta}$ of $K$ containing $\mathbf{Z}+\mathbf{Z} g$ as a submodule of index $f,[\Lambda: \mathbf{Z}+\mathbf{Z} g]=f$. Let $h(\Delta)$ denote the class number of locally principal ideals of $\Lambda$, and let $w(\Delta)$ denote the half of the cardinality of the unit group $\Lambda^{\times}$. Then

$$
b(s, f)=h(\Delta) / w(\Delta)
$$

Finally $c^{*}(s, f, q)$ is the number of non-equivalent embeddings of $K$ into $M_{2}\left(\mathbf{Q}_{q}\right)$ optimal with respect to $R_{q} / \Lambda_{q}$ such that $\psi(g) \in W_{N_{0}} R_{q}^{\times}$, i.e.

$$
c^{*}(s, f, q)=\left|\operatorname{Emb}\left(g, W_{N_{0}} R_{q}, R_{q} / \Lambda_{q}\right) / R_{q}^{\times}\right|
$$

in the notation of $[2,2.0]$.

1.4. For any $q \mid N, \operatorname{Emb}\left(g, R_{q}, R_{q} / \Lambda_{q}\right) / R_{q}^{\times}$can be given as follows ([2, Theorem 2.3]). Let $v=\operatorname{ord}_{q}(N)$ and $\rho=\operatorname{ord}_{q}(f)$. Put $\tilde{F}=\{\xi \in Z \mid$ $\Phi_{s}(\xi) \equiv 0\left(q^{v+2 e}\right)$ and $\left.2 \xi \equiv s\left(q^{e}\right)\right\}$. Let $F$ be a complete system of representatives of $F$ modulo $q^{v+e}$, and put $F^{\prime}=\left\{\xi \in F \mid \Phi_{s}(\eta) \equiv 0\left(q^{v+2 e+1}\right)\right\}$. Define $\psi_{\xi}: K \rightarrow M_{2}\left(\mathbf{Q}_{q}\right)$, by $\psi_{\xi}(g)=\left(\begin{array}{cc}\xi \\ -q^{-e} f(\xi) & s-\xi\end{array}\right)$ and $\psi_{\eta}^{\prime}: K \rightarrow M_{2}\left(\mathbf{Q}_{q}\right)$, by $\psi_{\eta}^{\prime}(g)=\left(\begin{array}{cc}s-\eta & -q^{-(v+e)} f(\eta) \\ q^{v+e} & \eta\end{array}\right)$ respectively. If $\left(q^{-2 \varrho}\left(s^{2}-4 n N_{0}\right)\right.$ is prime 
to $q$ (resp. divisible by $q$ ), the set $\left\{\psi_{\xi} \mid \xi \in F\right\}$ (resp. $\left\{\psi_{\xi} \mid \xi \in F\right\} \cup\left\{\psi_{\eta}^{\prime} \mid \eta\right.$ $\left.\left.\in F^{\prime}\right\}\right)$ is a complete system of representatives of $\operatorname{Emb}\left(g, R_{q}, R_{q} / \Lambda_{q}\right) / \tilde{R}_{q}^{\times}$. Let $c(s, f, q)$ denote its cardinality i.e. $c(s, f, q)=|F|$ (resp. $\left.|F|+\left|F^{\prime}\right|\right)$ if $q^{-2 e}\left(s^{2}-4 n N_{0}\right) \equiv 0 \quad($ resp. $\equiv 0) \bmod q$. If $\left(q, N_{0}\right)=1$, then $W_{N_{0}} R_{q}=R_{q}$. Hence $c^{*}(s, f, q)=c(s, f, q)$. We claim

Lemma. If $\left(q, N_{0}\right)>1$, and $q^{v} \| N_{0}$, then

$$
c^{*}(s, f, q)= \begin{cases}1, & \text { if } s \equiv 0 \quad\left(q^{v}\right) \text { and }(f, q)=1 \\ 0, & \text { otherwise. }\end{cases}
$$

Proof. Since $R_{q}^{\times}$normalizes $W_{N_{0}} R_{q}=\left(\begin{array}{cc}q^{v} Z_{q} & Z_{q} \\ q^{v} Z_{q} & q^{v} Z_{q}\end{array}\right), E=\operatorname{Emb}\left(g, W_{N_{0}} R_{q}\right.$, $\left.R_{q} / \Lambda_{q}\right) / R_{q}^{\times}$can be considered as a set of $\psi \in \operatorname{Emb}\left(g, R_{q} / \Lambda_{q}\right) / R_{q}^{\times}$such that $\psi(g) \in W_{N_{0}} R_{q}$. If $\psi_{\xi} \in E$, i.e. $\psi_{\xi}(g) \in W_{N_{0}} R_{q}$, then $\xi \equiv s-\xi \equiv 0\left(q^{v}\right)$. Since $\operatorname{det} \psi_{\xi}(g)=\xi(s-\xi)-f(\xi)=n N_{0}$, and $\operatorname{ord}_{q}\left(n N_{0}\right)=v$, we get $\operatorname{ord}_{q}(f$ $(\xi))=v$. Since $f(\xi) \equiv 0 \quad\left(q^{v+2 e}\right), \rho$ should be equal to 0 . Hence we can take $F=\{0\}$. Similarly if $\psi_{\eta}^{\prime} \in E$, we get $\operatorname{ord}_{q}(f(\eta))=v$. Then the condition $f(\eta) \equiv 0\left(q^{v+1}\right)$ for $F^{\prime}$, implies that $F^{\prime}$ is empty. Thus

$$
E=\left\{\begin{array}{l}
\{0\}, \quad \text { if } \quad s \equiv 0\left(q^{\nu}\right) \text { and } \rho=\operatorname{ord}_{q}(f)=0 \\
\text { empty, otherwise }
\end{array}\right.
$$

Hence $c^{*}(s, f, q)=|E|=1$ or 0 .

1.5. As is easily seen the case (p) occurs if and only if $N_{0}=4$. The case $(h)$ occurs if and only if $N_{0}$ is a square. Hence in the formula of $\operatorname{tr} T_{n} W_{N_{0}}$, the partial sum $\sum_{s}$ over the $s$ 's of type $(h)$ is given by

$$
\begin{aligned}
& -\sum_{s^{2}-4 n N_{0}=t^{2}} a(s) \Sigma b(s, f) \prod_{q \mid N} c^{*}(s, f, q) \\
= & -\varphi\left(\sqrt{N_{0}}\right) \cdot N_{0}^{k / 2-1} \sum_{a} a^{k-1} \prod_{q \mid N N_{0}^{-1}} c\left(s_{0}, f_{0}, q\right)
\end{aligned}
$$

where the sum $\sum$ is extended over $0<a<\sqrt{n}, a \mid n$ and $a^{2}+n \equiv 0(\bmod$ $\left.\sqrt{N_{0}}\right)$, and $s_{0}=\sqrt[a]{N_{0}}(a+n / a)>0, f_{0}=n / a-a, \varphi(m)$ denotes the Euler function. We remark that the volume part of the trace does not ap- 
pear since the intersection of the center $G L_{2}(\mathbf{Q})$ with $\Xi W_{N_{0}}$ is empty.

1.6. Summing up, we have obtained the following

Theorem. If $(n, N)=1$, the trace $\operatorname{tr} T_{n} W_{N_{0}}$ of the operator $T_{n} W_{N_{0}}$ on $S_{k}(N)$ is given as follows:

$$
\begin{aligned}
\operatorname{tr} T_{n} W_{N_{0}}= & -\frac{1}{2} \Sigma_{1} \prod_{q \mid N N_{0}^{-1}} c(s, f, q) \cdot \frac{h\left(\left(s^{2}-4 n N_{0}\right) f^{-2}\right)}{w\left(\left(s^{2}-4 n N_{0}\right) f^{-2}\right)} \cdot \frac{x^{k-1}-y^{k-1}}{x-y} \\
& -\delta_{1} \cdot \varphi\left(\sqrt{\left.N_{0}\right)} \cdot N_{0}^{k / 2-1} \cdot \Sigma_{2} a^{k-1} \cdot \prod_{q \mid N N_{0}^{-1}} c\left(s_{0}, f_{0}, q\right)\right. \\
& -\delta_{2} \cdot \frac{n^{\frac{k-1}{2}}}{2} \cdot \prod_{q^{\nu}|| N / 4}\left(q^{\left[\frac{v}{2}\right]}+q^{\left[\frac{v-1}{2}\right]}\right) \\
& +\delta_{3} \cdot \sum_{d \mid n} d,
\end{aligned}
$$

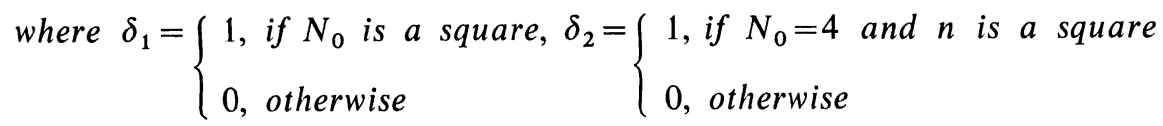

and $\delta_{3}=\left\{\begin{array}{l}1, \text { if } k=2 \\ 0, \text { otherwise. }\end{array} \quad\right.$ The sum $\Sigma_{1}$ is extended over the

pairs of integers $(s, f)$ such that $s^{2}<4 n N_{0}, s \equiv 0 \bmod N_{0}, f>0,\left(s^{2}-\right.$ $\left.4 n N_{0}\right) f^{-2} \equiv 0,1 \bmod 4$ and $\left(f, N_{0}\right)=1$. The sum $\Sigma_{2}$ is extended over the integers a such that $0<a<\sqrt{n}, a \mid n, a^{2}+n \equiv 0 \bmod \sqrt{N_{0}}$ and we put $s_{0}=\sqrt{N_{0}}(a+n / a), f_{0}=n / a-a$. The number $c(s, f, q)=|F|$ or $|F|+\left|F^{\prime}\right|$ hasb een explained in 1.4 .

2.1. Let $S_{k}\left(N, N_{0}\right)$ denote the space of cusp forms of weight $k$ for the group generated by $\Gamma_{0}(N)$ and $W_{N_{0}}$. Then $S_{k}\left(N, N_{0}\right)$ can be viewed as a subspace of $S_{k}(N)$. Let $S_{k}^{0}(N)$ be the essential part of $S_{k}(N)$, i.e. the space spanned by the new forms in the sense of [1] and put $S_{k}^{0}\left(N, N_{0}\right)=S_{k}^{0}(N) \cup S_{k}\left(N, N_{0}\right)$. Let $\operatorname{tr}_{N}\left(\right.$ resp. $\left.\operatorname{tr}_{N, N_{0}}, \operatorname{tr}_{N}^{0}, \operatorname{tr}_{N, N_{0}}^{0}\right)$ denote the trace on $S_{k}(N)$ (resp. $S_{k}\left(N, N_{0}\right), S_{k}^{0}(N), S_{k}^{0}\left(N, N_{0}\right)$ ). It is known (cf. [1]) that there exists a basis $\left\{f_{\lambda}\right\}$ of $S_{k}(N)$ such that each $f_{\lambda}$ is an eigenform of all the operators $T_{n}$ and $W_{q^{\nu}}\left(q \mid N_{0}\right)$, and $W_{N_{0}}$ maps $S_{k}^{0}(N)$ into itself. Since 


$$
f_{\lambda} \mid W_{N_{0}}= \begin{cases}f_{\lambda}, & \text { if } f_{\lambda} \in S_{k}\left(N, N_{0}\right) \\ -f_{\lambda}, & \text { if } f_{\lambda} \notin S_{k}\left(N, N_{0}\right) .\end{cases}
$$

Hence we obtain the following

\section{Proposition 1.}

$$
\begin{aligned}
& \operatorname{tr}_{N} T_{n}-2 \operatorname{tr}_{N, N_{0}} T_{n}=-N_{0}{ }^{k / 2-1} \cdot \operatorname{tr}_{N} T_{n} W_{N_{0}} \\
& \operatorname{tr}_{N}^{0} T_{n}-2 \operatorname{tr}_{N, N_{0}}^{0} T_{n}=-N_{0}{ }^{k / 2-1} \cdot \operatorname{tr}_{N}^{0} T_{n} W_{N_{0}}
\end{aligned}
$$

Remark. Let $g$ (resp. $g^{*}$ ) denote the gneus of the group $\Gamma_{0}(N)$ (resp. $\Gamma^{*}(N)=<\Gamma_{0}(N), W_{N}>$ ). Specializing our Theorem and Proposition 1 to the simplest case where $N_{0}=N, k=2$ and $n=1$, we get the well known formula of Fricke;

$$
g-2 g^{*}=\left\{\begin{array}{lc}
\frac{1}{2}(h(-4 N)+h(-N))-1, & \text { if }-N \equiv 1 \bmod 4 \text { and } N \neq 3, \\
\frac{1}{2} h(-4 N)-1, & \text { if }-N \equiv 0,2,3 \bmod 4 \text { and } \\
& N \neq 2,4 \\
0, & \text { if } N=2,3,4 .
\end{array}\right.
$$

2.2. For the $\operatorname{tr}_{N}^{0} T_{n} W_{N_{0}}$ given in Proposition 1, we have the following formula;

\section{Proposition 2.}

$$
\operatorname{tr}_{N}^{0} T_{n} W_{N_{0}}=\sum_{M \mid N N_{0}^{-1}} \beta\left(N N_{0}^{-1} M^{-1}\right) \cdot \sum_{Q^{2} \mid N_{0}} \mu(Q) \operatorname{tr}_{M N_{0} Q^{-2}} T_{n} W_{N_{0} Q^{-2}}
$$

where $\mu(m)$ denotes the Möbius function, and $\beta(n)=\sum_{d \uparrow_{n}} \mu(d) \mu\left(\frac{n}{d}\right)$.

Proof. Since $S_{k}(N)=\underset{M N|d| M^{-1}}{\bigoplus} S_{k}^{0}(M)^{d}$ (direct sum), where $S_{k}^{0}(M)^{d}=$ $\left\{f(d z) \mid f \in S_{k}^{o}(M)\right\}$. We have

$$
\operatorname{tr}_{N} T_{n} W_{N_{0}}=\sum_{\substack{M|N \\ d| N M^{-1}}} \operatorname{tr} S_{k}^{0}(M)^{d} T_{n} W_{N_{0}}
$$


Now for a divisor $M$ of $N$, assume $q^{\mu} \| M$ where $q$ is a fixed prime such that $q^{v} \| N_{0}$. Then by [1, Lemma 26], we get for any divisor $L$ of $N M^{-1}$ prime to $q, \operatorname{tr} W_{q^{\nu}}=0$ on $S_{k}^{0}(M)^{L^{q \delta}} \oplus S_{k}^{0}(M)^{L^{q \nu-\mu-\delta}}$ if $0 \leqq$ $2 \delta<v-\mu$, and for any other prime $q_{1}^{v^{1}} \| N_{0}$ distinct from $q$, $\operatorname{tr} W_{q 1}{ }^{{ }^{1}}$ on $S_{k}^{0}(M)^{L^{q^{\delta}}}=\operatorname{tr} W_{q_{1}^{\nu_{1}}}$ on $S_{k}^{0}(M)^{L^{q \nu-\mu-\delta}}$. Hence we get

$$
\operatorname{tr}_{N} T_{n} W_{N_{0}}=\sum_{M \mid N N_{0}^{-1}} d\left(\frac{N}{N_{O} M}\right) \Sigma_{\mu} \operatorname{tr}_{M Q_{\mu}}^{0} T_{n} W_{Q_{\mu}}
$$

where $d(m)$ is the number of divisors of $m$, and the sum $\Sigma_{\mu}$ is extended over all $\mu=\left(\mu_{1}, \ldots, \mu_{u}\right)$ such that $0 \leqq \mu_{i} \leqq v_{i}, \mu_{i} \equiv v_{i} \bmod 2(1 \leqq i \leqq u)$, and we put $Q_{\mu}=\prod_{i=1}^{u} q_{i}^{\mu_{i}}\left(u\right.$ : the number of prime factors of $\left.N_{0}\right)$. Put $N^{\prime}=$ $N N_{0}^{-1}, \lambda\left(N^{\prime}\right)=\operatorname{tr}_{N} T_{n} W_{N_{0}}$ and $\lambda^{0}(M)=\Sigma_{\mu} \operatorname{tr}_{M Q_{\mu}}^{0} T_{n} W_{Q_{\mu}}$ then

$$
\lambda\left(N^{\prime}\right)=\sum_{M \nmid N^{\prime}} d\left(\frac{N^{\prime}}{M}\right) \lambda^{0}(M)
$$

hence

$$
\lambda^{0}\left(N^{\prime}\right)=\sum_{M \mid N^{\prime}} \beta\left(\frac{N^{\prime}}{M}\right) \lambda(M)
$$

with $\beta(m)=\sum_{d \mid m} \mu(d) \mu\left(\frac{m}{d}\right)$.

While

$$
\begin{aligned}
\lambda^{0}\left(N^{\prime}\right) & =\sum_{\mu} \operatorname{tr}_{N^{\prime} Q_{\mu}}^{0} T_{n} W_{Q_{\mu}} \\
& =\sum_{\left.Q^{2}\right|_{N_{0}}} \operatorname{tr}_{N Q^{-2}} T_{n} W_{N_{0} Q^{-2}}
\end{aligned}
$$

hence

$$
\operatorname{tr}_{N}^{0} T_{n} W_{N_{0}}=\sum_{M \mid N^{\prime}} \beta\left(\frac{N^{\prime}}{M}\right) \sum_{Q^{2} \mid N_{0}} \mu(Q) \operatorname{tr}_{M N_{0} Q^{-2}} T_{n} W_{N_{0} Q^{-2}}
$$

3.1. Using our Theorem and Propositions we give here some numerical examples of the eigen-values of $T_{n}$ or $W_{N_{0}}$, exclusively for weight $k=2$. 
(1) The case $N=3 \cdot 5 \cdot 7=105, \operatorname{dim} S_{2}(105)=13$.

\begin{tabular}{l|rr|rr|rrrrrrr|rrr}
\hline \hline & \multicolumn{10}{|c|}{ eigen-values } \\
\cline { 2 - 12 } & \multicolumn{1}{|c|}{ I } & \multicolumn{1}{|c|}{ II } & \multicolumn{1}{|c|}{ III } & \multicolumn{2}{|c}{ IV } \\
\hline$W_{3}$ & 1 & 1 & -1 & -1 & 1 & 1 & 1 & -1 & -1 & -1 & 1 & 1 & -1 \\
$W_{5}$ & -1 & -1 & 1 & -1 & 1 & -1 & -1 & 1 & -1 & -1 & 1 & 1 & -1 \\
$W_{7}$ & 1 & -1 & 1 & 1 & -1 & 1 & 1 & -1 & 1 & 1 & -1 & -1 & -1 \\
$T_{2}$ & -1 & -1 & -1 & -1 & 0 & $\alpha$ & $\beta$ & 0 & $\alpha$ & $\beta$ & $\sqrt{5}$ & $-\sqrt{5}$ & 1 \\
\hline
\end{tabular}

$$
\left(\alpha, \beta=\frac{-1 \pm \sqrt{17}}{2}\right)
$$

I, II and III denote the old forms obtained from the forms of level $3 \cdot 5,3 \cdot 7$ and $5 \cdot 7$, respectively. IV denotes the new forms. Each column corresponds to an eigenform. From the above table, we can see, for example, $\operatorname{dim} S_{2}\left(\Gamma^{\prime}\right)=3$, for $\Gamma^{\prime}=<\Gamma_{0}(105), W_{3}, W_{5}>$.

(2) The case $N=7^{3}=343, \operatorname{dim} S_{2}^{0}\left(7^{3}\right)=24, \operatorname{dim} S_{2}^{0}\left(7^{3}, 7^{3}\right)=9$. The space $S_{2}^{0}\left(7^{3}\right)$ is divided into five blocks I, II, II ${ }_{\chi}$, III and IV of the eigen-spaces of Hecke operators and characteristic polynomial of $T_{p}$ in each block is as follows (The part left open in the table is not computed yet).

\begin{tabular}{|c|c|c|c|}
\hline & I & \multicolumn{2}{|c|}{ II } \\
\hline$T_{2}$ & $x^{3}+4 x^{2}+3 x-1=0$ & \multicolumn{2}{|c|}{$x^{6}+2 x^{5}-6 x^{4}-10 x^{3}+10 x^{2}+11 x-1=0$} \\
\hline$T_{8}$ & $x^{3}=0$ & \multicolumn{2}{|c|}{$x^{6}+5 x^{5}-x^{4}-34 x^{3}-28 x^{2}+49 x+49=0$} \\
\hline$T_{5}$ & $x^{3}=0$ & & \\
\hline \multirow[t]{2}{*}{$T_{11}$} & $x^{3}+9 x^{2}+20 x+13=0$ & & \\
\hline & $\mathrm{II}_{x}$ & III & IV \\
\hline$T_{2}$ & $\begin{array}{l}x^{6}+2 x^{5}-6 x^{4}-10 x^{3} \\
+10 x^{2}+11 x-1=0\end{array}$ & $\left(x^{3}-2 x^{2}-x+1\right)^{2}=0$ & $x^{8}-3 x^{2}-4 x+13=0$ \\
\hline$T_{3}$ & $\begin{array}{l}x^{6}-5 x^{5}-x^{4}+34 x^{8} \\
-28 x^{2}-49 x+49=0\end{array}$ & $x^{6}-20 x^{4}+124 x^{2}-232=0$ & $x^{3}=0$ \\
\hline$T_{5}$ & & $x^{6}-24 x^{4}+164 x^{2}-232=0$ & $x^{8}=0$ \\
\hline$T_{11}$ & & $\left(x^{3}-x^{2}-2 x+1\right)^{2}=0$ & $x^{3}-5 x^{2}-36 x+167=0$ \\
\hline
\end{tabular}


I and II form the space $S_{2}^{0}\left(7^{3}, 7^{3}\right)$, I and IV are corresponding to the Grössen-character of conductor 7 of $\mathbf{Q}(\sqrt{-7})$ (see [4]). Let $f(z)=$ $\Sigma a_{n} e^{2 \pi \mathrm{in} z} \in S_{2}^{0}\left(7^{3}\right)$, then $f_{x}(z)=\Sigma a_{n}\left(\frac{n}{7}\right) e^{2 \pi \mathrm{in} z}$ is also contained in $S_{2}^{0}\left(7^{3}\right)$ ([3] Prop. 3.64.) $\mathrm{II}_{x}$ is obtained from $\mathrm{II}$ in this way, i.e. if $f(z)$ is contained in II then $f_{\chi}(z)$ is contained in $\mathrm{II}_{x}$. Now we give some remarks for III. It seems that the part III is closely related to the theory of $[3,7.7]$ (Construction of class fields over real quadratic fields). If $f(z)$ is contained in III, $f_{\chi}(z)$ is also contained in III, and let $F$ (resp. $K$ ) be the field generated over $\mathbf{Q}$ by the eigen-values of $T_{n}$ for all $\left(\frac{n}{7}\right)=1$ (resp. for all $\left(\frac{n}{7}\right)=-1$ ) then we see $F=\mathbf{Q}\left(\zeta_{7}+\zeta_{7}^{-1}\right)$, where $\zeta_{7}=e^{\frac{2 \pi i}{7}}$ and $K$ is a quadratic extension of $F$ with relative discriminant $N_{F / Q} \mathfrak{d}(K / F)=29$. For $\left(\frac{p}{7}\right)=1 \quad\left(p:\right.$ a prime), let $p^{7}=\alpha_{p} \alpha_{p}^{\prime}$ where $\alpha_{p}$ is an element of the order of conductor 7 in $\mathbf{Q}(\sqrt{-7})$ normalized as $\left(\frac{\alpha_{p}+\alpha_{p}^{\prime}}{7}\right)=1\left(\alpha_{p}^{\prime}:\right.$ the conjugate of $\left.\alpha_{p}\right)$, for example $\alpha_{2}=\frac{13+7 \sqrt{-7}}{2}$. Let $a_{p}$ be an eigenvalue of $T_{p}$ and $\pi_{p}, \pi_{p}^{\prime}$ be the solutions of $x^{2}-a_{p} x+p \equiv 0(\bmod 29)$ in the prime field, then we can see

$$
\pi_{p}^{7}+\pi_{p}^{\prime 7} \equiv \alpha_{p}+\alpha_{p}^{\prime} \quad(\bmod 29)
$$

for $p=2,11$. Furthermore we may expect that $N_{F / Q} \mathrm{~d}(K / F)=29$ is related to the fact $N_{F / Q}\left(\varepsilon^{7}-1\right)=8 \cdot 29$ with $\varepsilon=\zeta_{7}+\zeta_{7}^{-1}$.

KYOTO UNIVERSITY

\section{References}

[1] A. O. L. Atkin, and J. Lehner, Hecke Operators on $\Gamma_{0}(m)$, Math. Ann. 185 (1970), 134-160.

[2] H. Hijikata, Explicit formula of the traces of Hecke operators for $\Gamma_{0}(N)$, to appear in J. Math. Soc. Japan.

[3] G. Shimura, Arithmetic theory of automorphic functions, Publications of Math. Soc. Japan, 1971.

[4] - On elliptic curves with complex multiplication as factors of the jacobians of modular function fields, Nagoya Math. J., 43 (1971), 199-208. 